\title{
Assessment of the severity of coronary artery disease at postmortem examination. Are the measurements clinically valid?
}

\author{
Jessica M Mann, Michael J Davies
}

\begin{abstract}
Objective-To compare the assessment of severity of coronary artery stenosis by the conventional pathology methods with a method designed to resemble quantitative angiography.

Design-31 human hearts harvested at necropsy were fixed by perfusion of the aortic root with $10 \%$ formol saline at 120 mm $\mathrm{Hg}$ for 24 hours. The right coronary and left anterior descending coronary arteries were transversely sliced every 2 $\mathrm{mm}$ and the absolute lumen dimensions plotted against the distance from the coronary ostium. Stenosis figures were calculated by comparing the lumen diameter with the lumen diameters in adjacent normal arterial segments in a manner identical to that used in angiographic measurement. The coronary artery segments were then processed histologically. Stenosis was then remeasured by comparing the lumen diameter with the diameter of the vessel within the internal elastic lamina identified by elastic van Gieson staining.

Results-Compared with the method that was analogous to angiography, the pathology method used on histological slides overestimated the degree of stenosis by $25-30 \%$. The lack of concordance between the methods was not a function of the severity of the stenosis.

Conclusion-When they read necropsy reports in which the severity of coronary artery stenosis is assessed cardiologists should be aware of the discrepancy between clinical and pathological methods.
\end{abstract}

(Br Heart f 1995;74:528-530)

Keywords: coronary stenosis quantification; coronary artery remodelling; coronary artery stenosis

British Heart

Foundation

Cardiovascular

Research Unit, St

George's Hospital

Medical School,

London

J M Mann

M J Davies

Correspondence to:

Dr J M Mann, BHF

Cardiovascular Research

Unit, St George's Hospital

Medical School,

Cranmer Terrace, London SW17 0RE.

Accepted for publication

26 April 1995 medicolegal issue of whether the managem of a patient should have included coronary artery bypass grafting. In forensic medicine the significance of coronary artery stenosis found
The increasing necessity for audit in the National Health Service has reawakened interdeaths after coronary artery bypass grafting. An aspect of these necropsies is to assess whether there is significant coronary artery at necropsy may have relevance to the cause of accidental and road traffic deaths.

At necropsy, stenosis is assessed by examining cross sections of the coronary arteries and comparing the lumen size with that of the area of the vessel at the same point. This comparison may be made visually or by formal measurement from a histological slide. The method used by pathologists is inherently different from that used in coronary angiography, where the reference point is the diameter of the angiographically normal lumen proximal to the segment of stenosis. Despite this fundamental difference in methods published papers and audit meetings often assume that the pathologists' assessment is equivalent to angiography. This paper compares the pathologist's method with a method designed to resemble quantitative angiography.

\section{Patients and methods}

We studied 31 hearts from patients who died suddenly from ischaemic heart disease. All hearts were fixed by perfusion with $10 \%$ formol saline at a constant pressure of $120 \mathrm{~mm}$ $\mathrm{Hg}$ for 24 hours. Once the hearts were fixed, the right coronary and left anterior descending coronary arteries were removed from the heart intact, cut transversely every $2 \mathrm{~mm}$, and their lumen size measured directly with an image analyser attached to a television camera (Analytical Medical Systems, Cambridge, UK). We excluded hearts with unusually large coronary arteries ( $>8 \mathrm{~mm}$ in diameter) and coronary artery segments containing fresh thrombus. The image of the coronary artery segment to be measured was visualised by a macro lens (Cannon) and projected onto the monitor of the image analyser by means of a video camera (JVC TK-870E). The lumen of the artery was then drawn by means of a mouse and a digitising pad, using a VIDS program. The measurements of the diameter of the lumen were then plotted against the distance from the arterial ostium to obtain plots analogous to those seen in quantitative angiography (fig 1). The degree of stenosis was defined as the relation between the dimensions of the lumen of a narrowed segment and those of the nearest normal proximal segment.

Once the macroscopic measurements had been taken, the coronary arteries were processed histologically. Histological sections $(6 \mu \mathrm{m})$ were stained with elastic van Gieson to identify the internal elastic lamina. The coronary artery sections were measured with the image analyser connected to an Olympus 
Figure 1 Graphic representation of the dimensions of the lumen of the right coronary artery as measured by the method that resembled quantitative angiography. The $y$ axis shows the diameter of the lumen of the artery measured in millimetres and the $x$ axis shows the distance from the ostium of the right coronary artery. Two areas of significant stenosis are present.

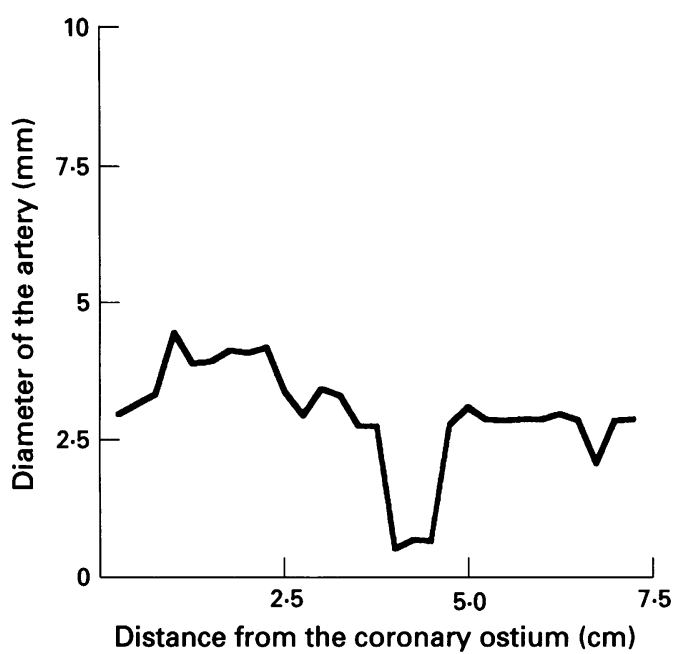

microscope, using the same video camera and imaging programme (VIDS) as previously described. The area of the lumen at the narrowed segment was compared with the area of the vessel within the internal elastic lamina in the same histological slide.

The two methods are inherently different. The first method resembles angiography. Lumen dimensions at different points in the vessel are used. In the second pathological method the lumen dimension is compared with overall vessel size at the same site in the vessel.

Statistical analysis of the data was performed with linear correlation curves and with the Bland and Altman method.

\section{Results}

There was a clear and statistically significant $(r$ $=0.78$ ) direct relation between the two methods (fig 2). None the less the pathology method gave overall a figure for diameter stenosis that was up to $30 \%$ higher than a method analogous to clinical angiography. The regression line cuts the vertical axis on which the histological stenosis is plotted at $29 \cdot 2 \%$. At any given level of "angiographic" stenosis, however, the histological method

\section{Figure 2 Graphic} representation of the severity of coronary artery stenosis as measured by both methods. The $y$ axis shows the histologically measured diameter stenosis, ranging from 0 to $100 \%$, and the $x$ axis shows the angiographically measured diameter stenosis. either overestimates or underestimates the degree of stenosis and it is not possible to use a simple correction factor. The spread of variation between the two methods is similar at all degrees of stenosis.

\section{Discussion}

This paper is concerned solely with indicating that the usual method used by pathologists to measure coronary artery stenosis overestimates, but can underestimate, the severity of the lesion, when compared with a method that follows the same principles that are used in clinical coronary angiography. Neither method is right or wrong, and both could give equally poor predictions of the haemodynamic significance of stenosis. This discrepancy in measurement needs to be borne in mind, however, when audit or medico-legal decisions are based on estimates of the severity of coronary artery stenosis by pathologists. In practice the discrepancies are likely to be even larger in routine necropsies. In the present study the arteries were perfused at pressure to distend the lumen and give a circular cross section. Without this initial procedure the arteries would be collapsed making measurements of the lumen even more inexact.

The discrepancies between coronary angiography and pathology have been a matter of concern for both cardiologists and pathologists. In 1965 Schwartz et al studied 25 hearts of patients who died after coronary artery bypass grafting. ${ }^{1}$ Although their evaluation was restricted to lesions resulting in $>75 \%$ stenosis, it did show that coronary angiography underestimates the degree of stenosis. Hutchins et al studied 28 patients with ischaemic heart disease who had had coronary angiography within 3 months of death; these 28 hearts provided them with 315 coronary artery segments. ${ }^{2}$ In $93 \%$ of segments the difference in the assessment of the degree of coronary artery stenosis was $50 \%$ or less, and in $68 \%$ of cases it was $25 \%$ or less. ${ }^{2}$ They concluded that the histological measurement overestimated the degree of stenosis, especially when the atherosclerotic plaque was eccentric.

The reasons for the overestimation when histological methods are used lie in the principle of remodelling of the vessel wall so well described by Glagov et al. ${ }^{3}$ When atherosclerosis develops, the vessel increases its external diameter to accommodate the developing plaque while simultaneously maintaining the dimensions of the lumen. This is why many plaques are angiographically invisible. There is a parallel increase in both the area of the atherosclerotic plaque and the size of the artery, until the plaque cross sectional area exceeds $40 \%$ of the area of the lumen. ${ }^{3}$ From this point remodelling can no longer maintain a totally normal lumen size but will continue and to some extent minimise the effect of further plaque growth. This process of arterial remodelling has recently been demonstrated in vivo by means of intravascular ultrasound. ${ }^{4}$ The histological method of measuring stenosis by using the diameter of the vessel at the site of a 
plaque as a reference point totally ignores this process of remodelling. Furthermore, when atherosclerosis becomes advanced, the media of the coronary artery undergoes focal atrophy, ${ }^{5}$ and this helps to preserve the size of the lumen by allowing the atherosclerotic plaque to "bulge" outwards.

Arterial remodelling as a process is poorly understood but involves rearrangement of medial smooth muscle cells. In some individuals it is prominent, in others scanty. Even in one individual remodelling can occur at one point but not at another. Despite all these caveats there is still a belief that measurements of coronary artery stenosis by histology at necropsy has great validity. ${ }^{6}$ On any particular histological section the method is reproducible with very small differences between repeated measurements. This does not, however, mean that the figures obtained are directly analogous to angiographic measurement in life or indeed that the figures have clinical relevance.

1 Schwartz JN, Kong Y, Hackel DB, Bartel AG. Comparison of angiographic and postmortem findings in patients with cor ang artery disease. Am ₹ Cardiol 1975;46:174-8.

2 Hutchins GM, Bulkley BH, Ridolfi RL, et al. Correlation of coronary arteriograms and left ventriculograms with coronary arteriograms and left ventriculogram

3 Glagov S, Weisenberg E, Zarins CK, Stankunavidius R, Kolettis GJ. Compensatory enlargement of human atherosclerotic coronary arteries. N Engl f Med 1987; 316:1371-5

4 Losordo DW, Rosenfield K, Kaufman J, Pieczek A, Isne JM. Focal compensatory enlargement of human arteries in response to progressive atherosclerosis. Circulation 1994;89:2570-7.

5 Isner JM, Donaldson RF, Fortin AH, Tischler A, Clarke RH. Attenuation of the media of coronary arteries in advanced atherosclerosis. Am $\mathcal{F}$ Cardiol 1986;58:937-9.

6 Corrado D, Basso C, Poletti A, Angelini A, Valente M, Thiene G. Sudden death in the young. Is acute coronary thrombosis the major precipitating factor? Circulation 1994;90:2315-23. 